\title{
Comparison of the Flux Reversal and VernierHybrid Machine for a Hinged Wave Energy Converter
}

\author{
Lewis Chambers \\ School of Engineering \\ Newcastle University \\ Newcastle Upon Tyne, UK \\ 1.n.chambers2@newcastle.ac.uk
}

\author{
Edward Spooner \\ Fountain Design Limited \\ Shildon, UK
}

\author{
Nick J. Baker \\ School of Engineering \\ Newcastle University \\ Newcastle Upon Type, UK
}

\author{
Mike Galbraith Fountain \\ Design Limited Shildon, UK \\ mikeg@fountaindesign.co.uk
}

\begin{abstract}
This paper compares two variable reluctance permanent magnet machines - a flux reversal and Vernier hybrid variant. The machine, which is being developed in conjunction with Mocean Energy, is required to operate at very low speed. The paper describes the application and electrical topology development, with a focus on the optimization routine used. Results show there is a conflict between peak torque and cogging torque, but also that the peak induced voltage and cogging torque are highly dependent on and sensitive to the rotor tooth width, rather than if the machine is Vernier or flux reversal type.
\end{abstract}

Index Terms - wave energy, Vernier hybrid, flux reversal

\section{INTRODUCTION}

Wave energy is a potentially huge source of renewable energy which is yet to reach commercial maturity. Among the many challenges faced by the technology is the need for the development of a bespoke electrical power train. Waves are slow which has led to a range of novel electrical generators being developed for a range of wave energy converters. One such concept being developed for full scale is a floating wave energy device consisting of two rigid bodies connected via a single hinged joint [1]. Power take off is via the relative movement felt at the hinge. The peak speed of movement is equivalent to around $1 \mathrm{rpm}$, very challenging for the electrical machine, which has led to machines with inherent magnetic gearing being considered. The large scale and harsh marine environment imply a robust topology is preferred, and experience from the developer implies a mechanical gearbox is problematic.

The developer in this project is Mocean Energy, and the work contained within this paper pertains to their product 'Blue Star', a hinged wave energy converter designed to fit inside a $40 \mathrm{ft}$ shipping container and supply renewable power to sub-sea applications - from autonomous ROVs and underwater vehicles, to sub-sea control systems for the oil and

This work was supported by the Engineering and Physical Sciences Research Council (grant number EP/S024069/1). simulation work was
As presented at 2021 IEEE Energy Conversion Congress and Exposition (ECCE). 2021 conducted within the Centre for Doctoral Training in Sustainable Electric Propulsion. The practical work was part funded an InnovateUK smart award, reference 56808 .

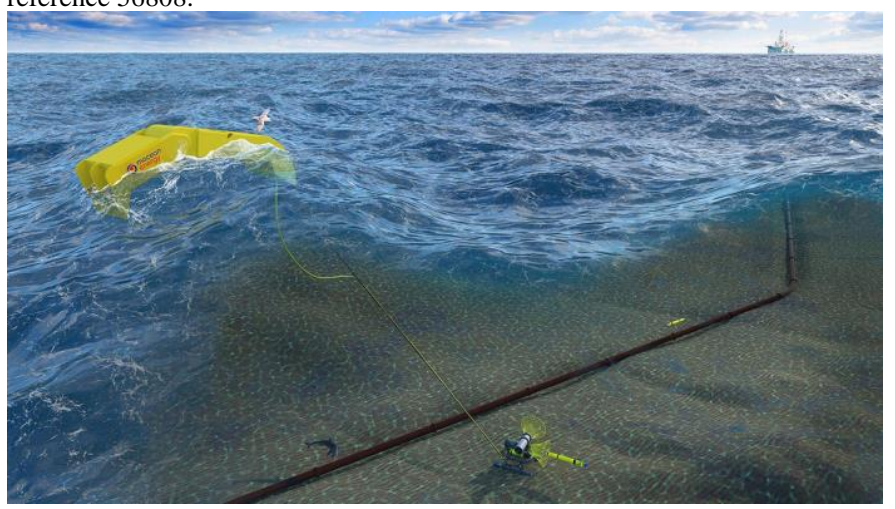

Fig. 1: Mocean Blue Star concept art [1].

gas industry. Although the device is designed for supplying power in sub-sea applications, the findings in this research are applicable to all multi-body hinge based wave energy converters, such as the 'Oyster' [2], or Mocean's 'Blue Horizon' [1].

A laboratory version of the electrical machine developed for this application is shown in Fig. 2. It is a Vernier Hybrid Machine (VHM) [3], developed for its high shear stress and inherent magnetic gearing. In this paper the conflict between peak torque, torque ripple and cogging torque is used to evaluate two alternative variants of this machine to be built at a larger scale.

\section{Flux Reversal Machine AND VerniER Hybrid MACHINE}

Generally, Variable Reluctance Permanent Magnet (VRPM) machines are characterized by a series of magnets of alternating polarity and short pitch, permeated by toothed iron poles, providing rapid reversal of flux. There is some variation between topologies in terms of orientation and placement of 


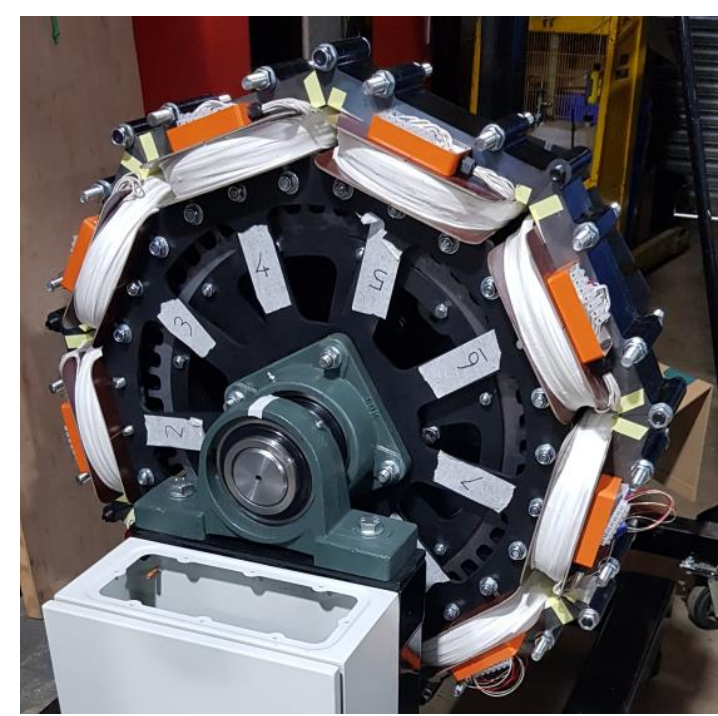

Fig. 2: Laboratory prototype of the vernier hybrid machine.

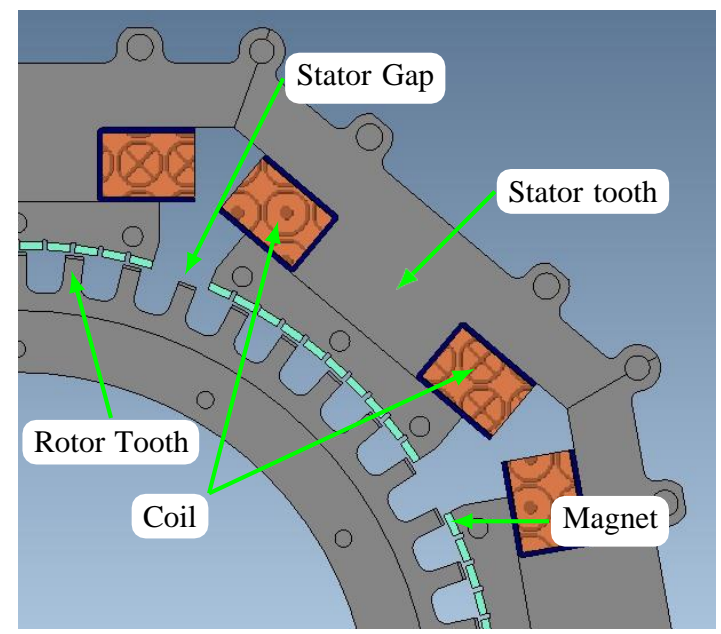

Fig. 3: FEA model of the vernier hybrid machine used for verification.

magnets and coils and relative size of rotor tooth pitch and magnet pitch [3], [4]. Figs. 2 and 3 show a topology with 10 magnets mounted on each stator tooth, encompassed by a single tooth winding. The rotor consists of regular teeth and slots to modulate the field. In this example, 9 stator teeth are formed into 3 phases.

Two topologies in this family are the Flux Reversal Machine (FRM), where number of magnet and rotor pitches are equal, and the Vernier Hybrid Machine (VHM), where they are not. In an FRM, all magnets in a single phase share the same relative position with the nearest rotor tooth - Fig. 5a. This results in the maximum possible peak flux linkage and hence gives the machine with the highest back emf and greatest peak torque. Unfortunately, it also means that the component of cogging torque associated with each magnet is in phase across the stator tooth, potentially causing problems for the power train in no load operation.

The VHM stems from the Vernier Machine (VM) and stands
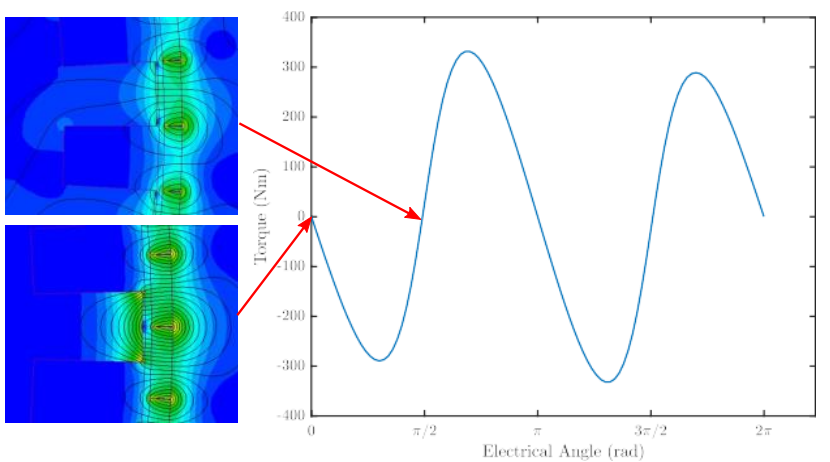

Fig. 4: Torque waveform for a flux reversal machine with one phase active and the rotor positions marked.

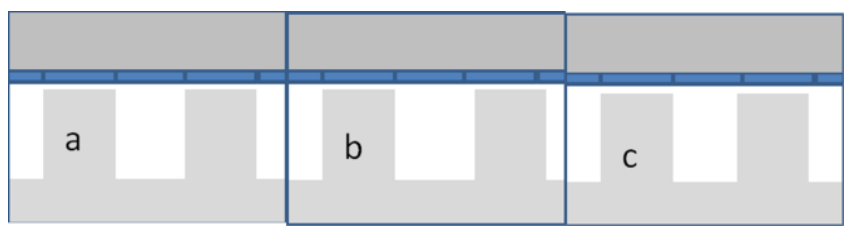

Fig. 5: Relative tooth and magnet positions for a Flux Reversal (a), and Vernier Hybrid (b \& c) variant. In all variants, the left-hand tooth is fully aligned with the magnet. In (c) the rotor pitch is less than the stator pitch, hence there is a higher number of rotor poles and a greater overlap of teeth and the stator pole.

apart by moving the magnets from the rotor, onto the stator, and utilises concentrated windings instead of distributed. In essence, it contains elements of the FRM and VM, hence the 'Hybrid' part of the name.

In the VHM, the number of rotor pitches does not equal that of the magnet, but instead is defined as:

$$
p_{r}=p_{m} \pm p_{c}
$$

Where $p_{r}$ is the number of rotor poles, $p_{m}$ is the number of magnet poles, and $p_{c}$ is the number of coil poles.

This relationship gives two possible designs that will form a part of this study. These are termed $\operatorname{VHM}(-)$ and $\operatorname{VHM}(+)$, where the symbol signifies the sign taken in (1) such that VHM(-) has less rotor pole pairs than magnet pole pairs, and $\operatorname{VHM}(+)$ has more. The $\operatorname{VHM}(+)$ has been shown to have a poorer induced voltage and hence loaded torque performance [5], however, it will be included in the study for the sake of fairness and comparison.

\section{Cogging Torque and Harmonics}

Consider a single tooth passing over a magnet pair with no stator current. For each electrical cycle there are 4 positions of zero resultant torque, corresponding to full alignment with a magnets (d-axis) and full misalignment (q-axis), where the centre of the tooth is aligned with the point where magnets touch, see for example [6]. The first two points are shown in Fig. 4

The cogging torque can be approximated to the sum of a fundamental and a second harmonic. For a three phase FRM, 
adjacent stator teeth are separated by a multiple of magnet pole pitch / 3 . Hence the resultant cogging torque for the whole machine is the sum of three waveforms shifted by 120 electrical degrees. The fundamental and the second harmonic cancel out and so the idealized cogging waveform would result in a zero cogging force three phase machine. Unfortunately, in reality higher order harmonics exist in the cogging profile and any multiple odd harmonics are present in the full machine. Further, the triplen harmonics in each phase can combine to a magnitude three times larger. Experience shows that FRMs are likely to have large cogging forces even in three phase variants.

To avoid this, the vernier variant was developed [7], whereby the rotor pitch and stator pitch are not equal equivalent to short pitching a winding in a conventional electric machine. The effect can be achieved by increasing (Fig. 5b) or decreasing (Fig. 5c) the rotor pitch relative to the magnet pitch. Both variants have the potential for reduced cogging, as the total across a stator tooth is now the sum of six individual interactions separated by a small phase difference. Introduction of the vernier concept necessarily reduces the peak magnet flux cutting the coils as individual magnets in a single phase no longer align with a rotor tooth at the same time. In theory this should mean that in selecting a FRM or VHM there is a design trade-off between cogging torque and peak torque capability. However, in practice, the patterns of alignment and misalignment in the VHM introduce additional rotating harmonics into the air gap, some of which do useful work and improve the performance of the VHM.

\section{METHODOLOGY}

This paper seeks to make a fair and direct comparison between the FRM and VHM topologies using Finite Element Analysis (FEA) and verified by experimental results. The FRM requires a $120^{\circ}$ spacing between phases in order to function, but the VHM designs do not. Therefore, a FRM cannot operate using a VHM stator, but a VHM can operate using a FRM stator.

Herein lies an issue however, because if a FRM and VHM were designed using a spacing of 1.3 magnet pitches between the stators, the VHM(-) would not be feasible as the three phases cancel out. For the purposes of this paper, it is necessary to use different stator spacings for the two machine types. A side-effect of this is that there will be a slight difference in number of magnet pole pairs in the air gap circumference.

The topologies investigated have a laminated stator with 9 teeth bearing 10 magnets of alternating polarity, wound by a single coil per tooth. Each tooth is separated by 1 and 1.3 magnet pitches in the VHM and FRM designs respectively this is the 'stator gap' found in Fig. 3. In total, each machine has 5 pole pairs on each of the 9 stator teeth, making 45 pole pairs from the magnets, plus 1 or 1.3 pole pairs in each of the 9 stator tooth gaps. This makes a total 54 and 57 magnetic pole pairs in the VHM and FRM respectively. In all topologies there are 3 coil pole pairs, and the rotor is a slotted and laminated iron structure. The FRM has 57 rotor pole pairs and the VHM
TABLE I: Machine topologies used in study.

\begin{tabular}{|c|c|c|c|}
\hline & FRM & $\mathrm{VHM}(+)$ & VHM(-) \\
\hline Stator teeth & \multicolumn{3}{|c|}{9} \\
\hline Magnets per stator tooth & \multicolumn{3}{|c|}{10} \\
\hline Magnet pitches between stator teeth & 1.3 & \multicolumn{2}{|c|}{1} \\
\hline Coil pole pairs & \multicolumn{3}{|c|}{3} \\
\hline Magnet pole pairs & 57 & \multicolumn{2}{|c|}{54} \\
\hline Rotor pole pairs & & 57 & 51 \\
\hline Outer rotor radius $(\mathrm{mm})$ & \multicolumn{3}{|c|}{307.5} \\
\hline Airgap length (mm) & \multicolumn{3}{|c|}{2} \\
\hline Magnet thickness (mm) & \multicolumn{3}{|c|}{5} \\
\hline Rotor slot depth (mm) & \multicolumn{3}{|c|}{26} \\
\hline Turns per coil & \multicolumn{3}{|c|}{1300} \\
\hline Lamination thickness (mm) & \multicolumn{3}{|c|}{2} \\
\hline Axial length (mm) & \multicolumn{3}{|c|}{100} \\
\hline
\end{tabular}

TABLE II: Additional dimensions of constructed machine.

\begin{tabular}{|c|c|}
\hline Dimension & Value \\
\hline Magnet width $(\mathrm{mm})$ & 15 \\
\hline Magnet length $(\mathrm{mm})$ & 50 \\
\hline Wire gauge $(\mathrm{mm})$ & 1.25 \\
\hline Insulation thickness $(\mathrm{mm})$ & 3 \\
\hline Rotor tooth width $(\mathrm{mm})$ & 12 \\
\hline
\end{tabular}

rotor pole pairs are found from (1) to be 51 or 57 for the $\operatorname{VHM}(-)$ and $\mathrm{VHM}(+)$ respectively. The full specifications of the machines can be found in Table I.

In the FEA study, elements of the machine geometry were parameterised into a series of ratios. For example, the rotor tooth ratio is the rotor tooth width as a fraction of the rotor pitch. For all designs, the ratios were optimised for peak induced voltage.

\section{MODEL VALIDATION}

For this project, the VHM(-) has been built by Foundation Design Limited and was used for the purpose of validating the FEA models. Further dimensions specific to the constructed design can be found in Table II, and the materials used can be found in Table III.

The machine was built with modularity in mind. This can be seen best in Fig. 3. The stator is separated into 9 separate modules comprising of one tooth, 10 magnets, and a single concentrated coil, all of which are bolted together. Building the machine in this fashion helps to negate the common wave energy converter concern of maintenance; if there is an breakdown, a single module can be swapped out for repair.

However, this introduced a small issue. The stator laminations were manufactured from cold rolled steel and cut via laser. The laser cutting method did not make a clean and straight cutting edge, but rather an hourglass shaped edge. Thus the stator modules did not pair flush. Further processing via mill was required, as a result, the stator is not entirely uniform around the circumference. The ramifications of this will become more apparent in the results section.

During this case study, a physical VHM was constructed to verify the FEA model. Both were run at $0.5159 \mathrm{rad} / \mathrm{s}$ and the result of one electrical cycle is displayed in Fig. 6a. As mentioned in Section IV, there were some issues during manufacture producing slight deviations in the stator 
TABLE III: Materials used in machine construction.

\begin{tabular}{|c|c|}
\hline Component & Material \\
\cline { 1 - 1 } Rotor & DD11 steel \\
\cline { 1 - 2 } Stator core & N42H \\
\cline { 1 - 2 } Magnets & Ni Cu black epoxy \\
\hline Adhesive & Tufnol \\
\hline Coils & 'Magnabond' CAB200 copper \\
\hline Insulation & Tufno \\
\hline
\end{tabular}

modules from the designed geometry. This, combined with small fluctuations in the rotor speed led to variation in the peak induced voltage in the experimental machine. Electrical cycles with the maximum and minimum peak amplitude are plotted with the FEA results to give proper comparison.

It should be noted here that the waveforms do not end at the same time in Fig. 6a because the rotor speed was not constant through the experimental measurement. This is problematic because the voltage is proportional to the rotor speed. For fairness in the comparison, all 3 waveforms were scaled to $1 \mathrm{rad} / \mathrm{s}$ by dividing the voltages by their respective speeds, and plotted against the electrical angle across one cycle, shown in Fig. 6b.

With the voltages scaled and normalised it is easier to make comparisons. There is still some variation between the minimum and maximum experimental output, this can be attributed to the eccentricity of the stator modules. The peak values are $147.15 \mathrm{~V}$ and $136.14 \mathrm{~V}$ for the simulated and maximum experimental respectively, this is an error of $7.48 \%$ on the peak induced voltage. It is hence used to compare the VHM and FRM variants of this machine with good confidence.

\section{Simulated COMPARISON OF FRM AND VHM}

Three FEA models were used to make a full comparison between the flux reversal and vernier hybrid machine. They are compared here, first in terms of induced voltage and cogging torque, and thereafter in terms of loaded thrust torque. Details of the models can be found in Table I.

Before the results are discussed, a brief explanation of the parameterised ratios should be made. Two ratios are used in the analysis. The rotor tooth ratio is the rotor tooth width divided by the rotor pitch width and the magnet fill ratio is the magnet width divided by half the magnet pitch width - this accounts for one magnet pitch containing two magnets. The magnet fill ratio is explained here, but it is set to unity and is not varied as it is beyond the scope of this study. In this section, the rotor tooth ratio is set to ensure that the rotor tooth width is equal to the width of one magnet. These were $0.5035,0.4755$, and 0.5315 for the FRM, VHM(-), and $\operatorname{VHM}(+)$ respectively.

\section{A. Unloaded}

To assess the unloaded performance of the three topologies, they were simulated via FEA at a constant speed of $1 \mathrm{rad} / \mathrm{s}$ for one electrical cycle.

Fig. 7 shows the unloaded induced voltage normalised across one electrical cycle, here it is immediately apparent that the FRM and $\operatorname{VHM}(-)$ are the superior topologies, peaking at

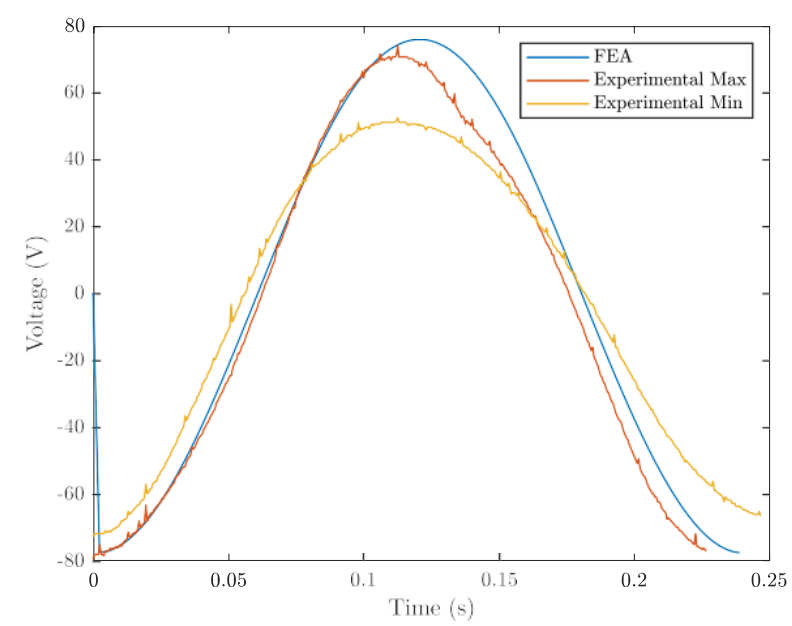

(a)

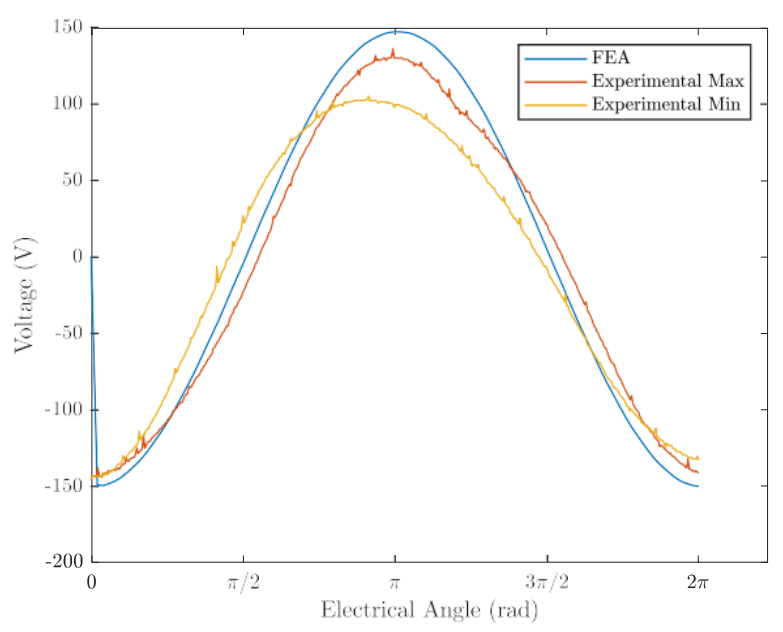

(b)

Fig. 6: FEA model compared against the maximum and minimum peak induced voltage from experimental data across all coils. (a) shows the raw voltage output. (b) shows the voltage output scaled to $1 \mathrm{rad} / \mathrm{s}$ and normalised across one electrical cycle.

141.95 V and 142.83 V respectively. The VHM(+) on the other hand is quite lower, at $116.84 \mathrm{~V}$.

The FRM was expected to be the highest as explained in Section II, because in a single phase, all magnets share the same relative position to the rotor teeth. As such, all magnets in a phase reach the peak flux position simultaneously, hence giving a large peak induced voltage.

Following this logic, the VHM designs should have a lower voltage amplitude as the magnets in a phase reach the peak flux position in an offset pattern. In practice, this is true, but having a rotor pole number different to the magnet pole number has the additional effect of introducing new harmonics into the air gap. Some of these harmonics can help to boost the VHM voltage induction. In the case of the $\operatorname{VHM}(-)$, it is able to compensate the alignment loss and compete with the FRM. The $\operatorname{VHM}(+)$ however, develops little gains. 


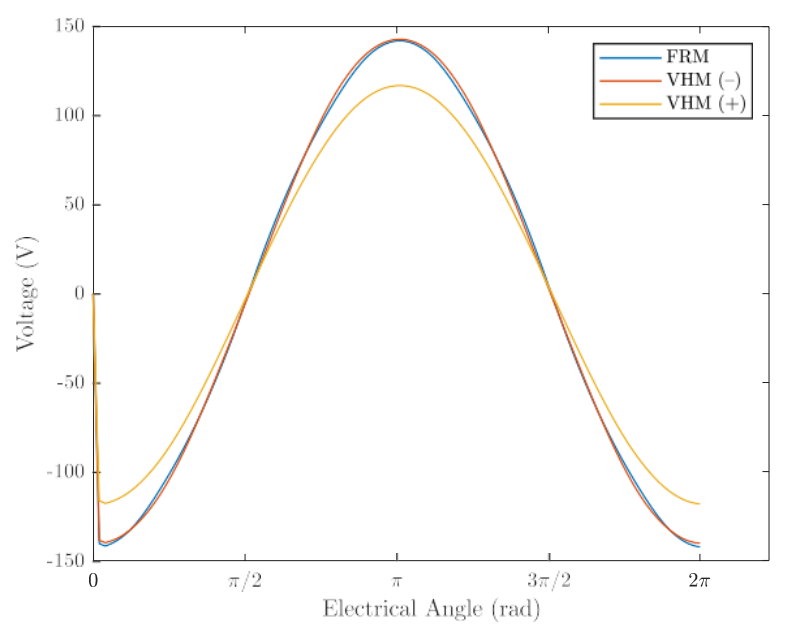

Fig. 7: Induced voltage into one coil with no load applied.

Moving on to the cogging torque, in Fig. 8, it can be seen that the previous description of the FRM holds true. There is a strong $6^{\text {th }}$ harmonic dominating the waveform at a $40.1 \mathrm{~N} \mathrm{~m}$ magnitude. A side-effect of maximum magnetic alignment is a correspondingly large alignment force resisting further motion.

The VHM topologies on the other hand experience lower cogging torques, $21.68 \mathrm{~N} \mathrm{~m}$ and $9.49 \mathrm{~N} \mathrm{~m}$ for the $\operatorname{VHM}(-)$ and $\mathrm{VHM}(+)$ respectively. The previously mentioned misalignment of rotor teeth causes the torque from individual tooth/magnet interactions to partially cancel out, especially in the $\operatorname{VHM}(+)$ where the cogging torque is lowest. This leaves behind a mixture of a $3^{\text {rd }}$ and a $6^{\text {th }}$ harmonic.

This is a common compromise in direct drive machines, there is typically an inverse relationship between the electromagnetic performance and cogging torque. In wave energy converters this compromise cannot be taken lightly. In the case of the floating hinged device considered in this study, if the magnetic alignment force is too strong, in low sea states, the device may just bob up and down like a buoy instead of rotating at the hinge to develop power.

\section{B. Loaded}

The designs were also simulated at $1 \mathrm{rad} / \mathrm{s}$ under loaded conditions of $2 \mathrm{~A}_{\mathrm{rms}} / \mathrm{mm}^{2}$ for one electrical cycle. This is shown in Fig. 9 and follows the pattern of the unloaded results. The FRM and $\operatorname{VHM}(-)$ lead with a mean load torque of $2548 \mathrm{~N} \mathrm{~m}$ and $2519 \mathrm{~N} \mathrm{~m}$ respectively, while the $\mathrm{VHM}(+)$ trails behind at $2034 \mathrm{~N} \mathrm{~m}$. It can be seen then, that the VHM can compare to the FRM but with a lower cogging torque.

Focusing on the torque ripple. They are $11.48 \%, 12.04 \%$, and $12.09 \%$ for the FRM, $\operatorname{VHM}(-)$, and $\operatorname{VHM}(+)$ respectively. The FRM operates will a lower torque ripple, but not significantly so.

Going on from here, the VHM(+) will no longer be considered in the analysis and focus will be placed on the FRM and VHM(-) instead.

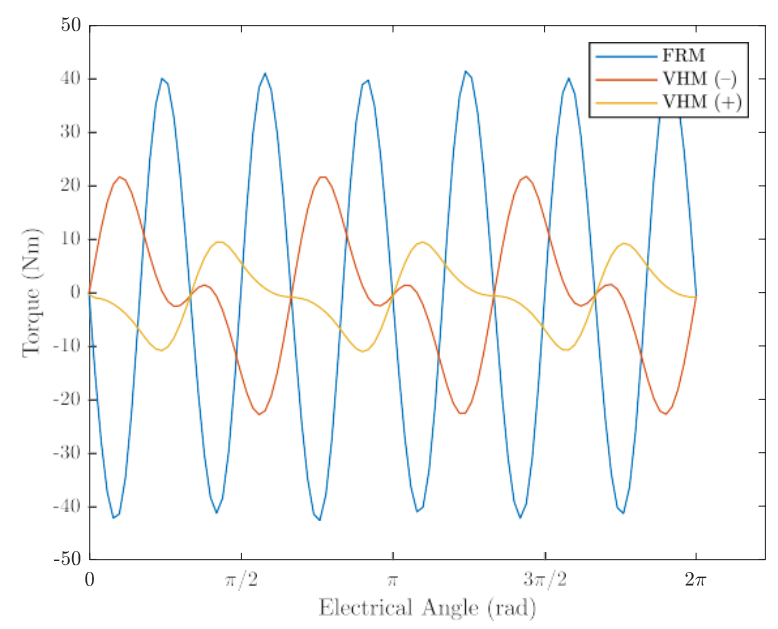

Fig. 8: Cogging torque produced with no load applied.

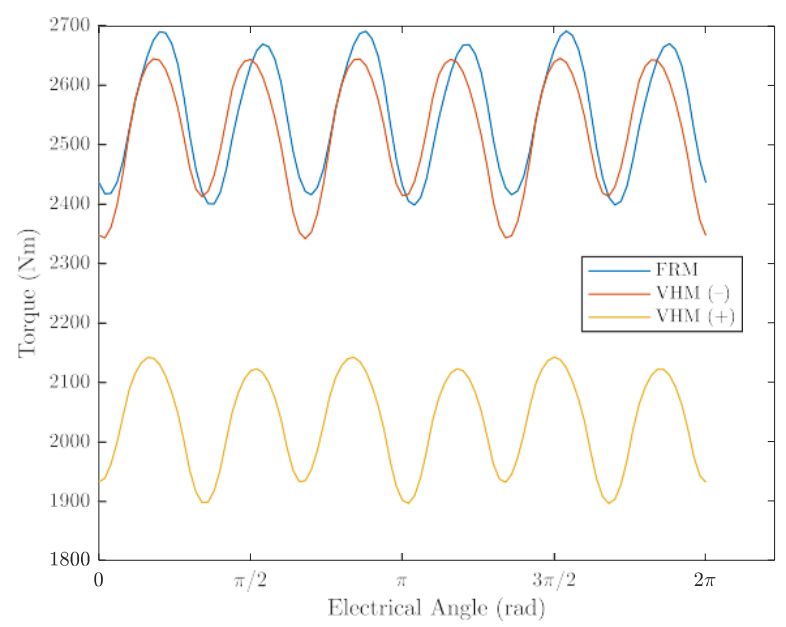

Fig. 9: Thrust torque at a load of $2 \mathrm{~A}_{\mathrm{rms}} / \mathrm{mm}^{2}$.

\section{Rotor Tooth Sensitivity}

In the previous sections the rotor tooth ratio was set separately to fix the rotor width to the width of a single magnet. In this section the rotor tooth ratio was varied from 0.1 to 0.7 in steps of 0.025 for the FRM and $\operatorname{VHM}(-)$. Fig. 10 shows the results, on the left $y$-axis is the cogging torque, showing the maximum and minimum peaks at each value of rotor tooth ratio. On the right $y$-axis is the peak voltage at each value.

First looking at the peak voltage, each topology follows a sensible pattern. Starting at the low end, the peak voltage increases with rotor tooth ratio, reaching a maximum of $187.76 \mathrm{~V}$ at 0.2 in the FRM and $178.95 \mathrm{~V}$ at 0.275 in the VHM. After this point the voltage gradually declines as the tooth width approaches and overtakes the width of one magnet, resulting in leakage flux across the tooth. It is apparent that even with the additional harmonics of the VHM, the FRM is still superior in terms of maximum possible voltage. 


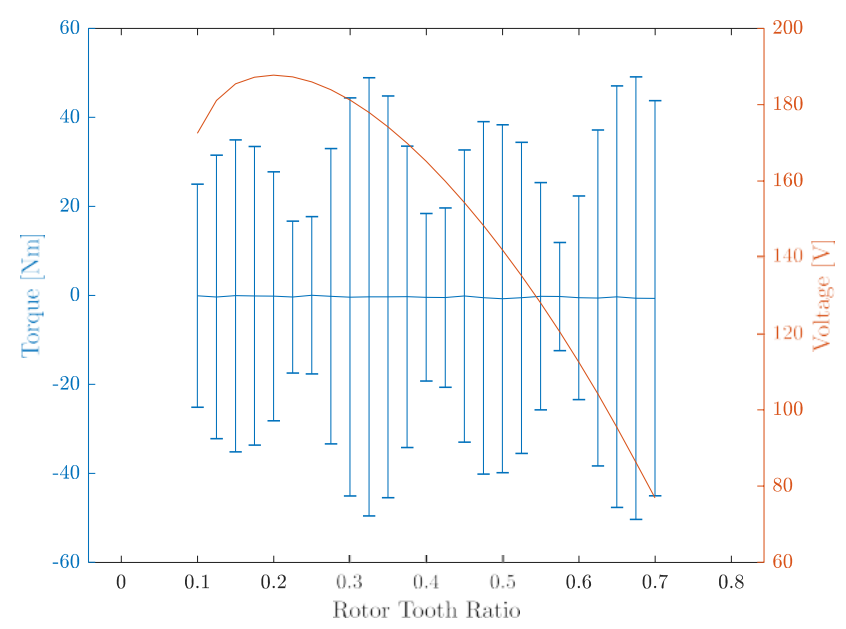

(a) Flux reversal machine.

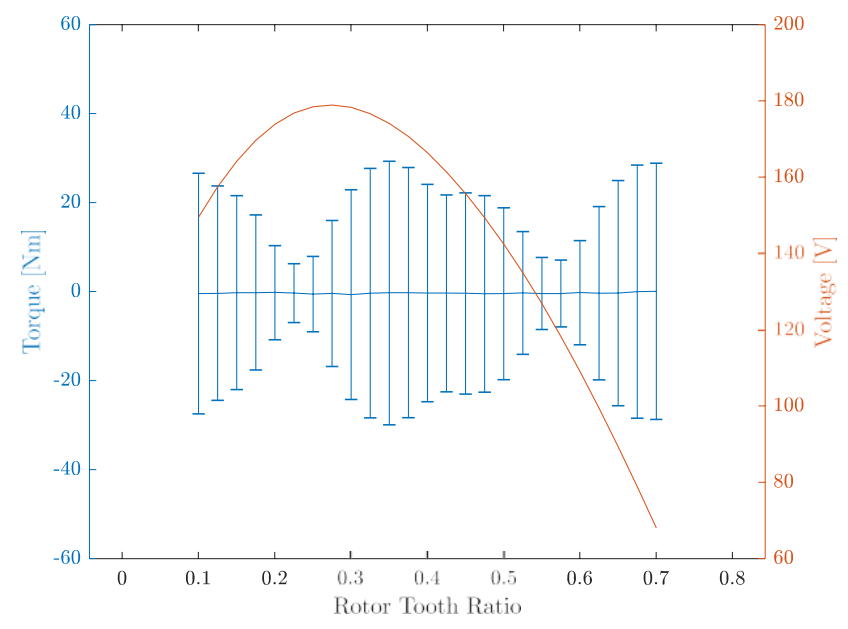

(b) Vernier hybrid machine.

Fig. 10: Cogging torque maximum and minimum points plotted together with peak induced voltage for the (a) flux reversal machine and (b) vernier hybrid machine.

The cogging torque on the other hand is more difficult to analyse. In both topologies there are several peaks and troughs in the maximum cogging torque, clearly the story told earlier is only true at that rotor tooth ratio. Such strong variation in cogging torque across the range of rotor tooth ratios demonstrates that the topologies are highly sensitive to changes in rotor tooth width and the full range should be considered when comparing the two directly. Simply comparing them at one ratio is insufficient to tell to whole story, and in fact may even be misleading. Regardless, using these plots it is simpler to select a design of desirable voltage and cogging torque.

It can be summed up here that across a wide range of values, the VHM has a comparable voltage to the FRM, with a lower cogging torque, but the FRM has the highest possible induced voltage.

\section{Cogging Harmonics and Tuning}

The FRM and VHM are both machines with three phases. Multiple of three harmonics do not cancel out and are summed instead. In the topologies shown, these harmonics make up the contents of the cogging torque.

This concept can be easily demonstrated through harmonic analysis. Fig. 11 shows the harmonics for the FRM machine across the range of rotor tooth ratio values given in Section VI-C. The waveforms across the range can be approximated to a $3^{\text {rd }}$ and $6^{\text {th }}$ harmonics, all other harmonics have either cancelled out or are insignificant.

The content of the waveforms can be better understood in this form. Each harmonic has peaks and troughs across the range of values, but it can be noted that at each trough the polarity of the phase angle reverses. This indicates that the direction of torque is switching at these points.

Fig. 12 shows a third and sixth harmonic waveform with an example rotor tooth drawn on. If that tooth were to move right; the left side will experience an increasingly positive attraction force from the $3^{\text {rd }}$ harmonic, and the right side will experience an equal but negative attraction force. The two forces on the tooth cancel out completely and the harmonic contributes no cogging torque. This is the case in the $3^{\text {rd }}$ harmonic when the rotor tooth ratio is equal to 0.0175 and 0.5 for the FRM, the harmonic is effectively tuned out and contributes no cogging torque. Unfortunately, in the same figure it can be seen that when moving right, the left and right side of the tooth are subjected to equally positive attraction force from the $6^{\text {th }}$ harmonic. Hence, for this tooth, when the $3^{\text {rd }}$ harmonic is tuned out, the $6^{\text {th }}$ harmonic is maximised.

So then, it is entirely possible for a designer to tune out harmonics as required by the design. For example, in the original FRM and VHM comparison, the FRM design fully tuned out the $3^{\text {rd }}$ harmonic but left behind a very large $6^{\text {th }}$ harmonic and appeared to have a much worse cogging torque.

\section{CONCLUSION}

In this paper the FRM and VHM topologies have been compared for application in a hinged wave energy converter. The two topologies were compared with the rotor tooth width equal to the width of one magnet. Unloaded and loaded simulations demonstrated that the $\operatorname{VHM}(-)$ was able to compete with the FRM, while the $\mathrm{VHM}(+)$ performed poorly.

It was further revealed that the rotor tooth width has a very high sensitivity on the cogging toque harmonic content and magnitude. The selection of rotor tooth is more significant than the decision between flux reversal and Vernier variants of this machine. Because of this, it is misleading to compare the designs for one rotor tooth ratio. It was shown that the FRM has the highest potential voltage, but across most values of rotor tooth ratio the $\operatorname{VHM}(-)$ has a competitive induced voltage and with a lower cogging torque. Afterwards it was found that the cogging torque can be approximated as a $3^{\text {rd }}$ and $6^{\text {th }}$ harmonic, and that by altering the rotor tooth width, these harmonics can be actively tuned out at the liberty of the designer.

In this paper, the main variable of alteration has been the rotor tooth ratio, and it has proven to be a highly sensitive 

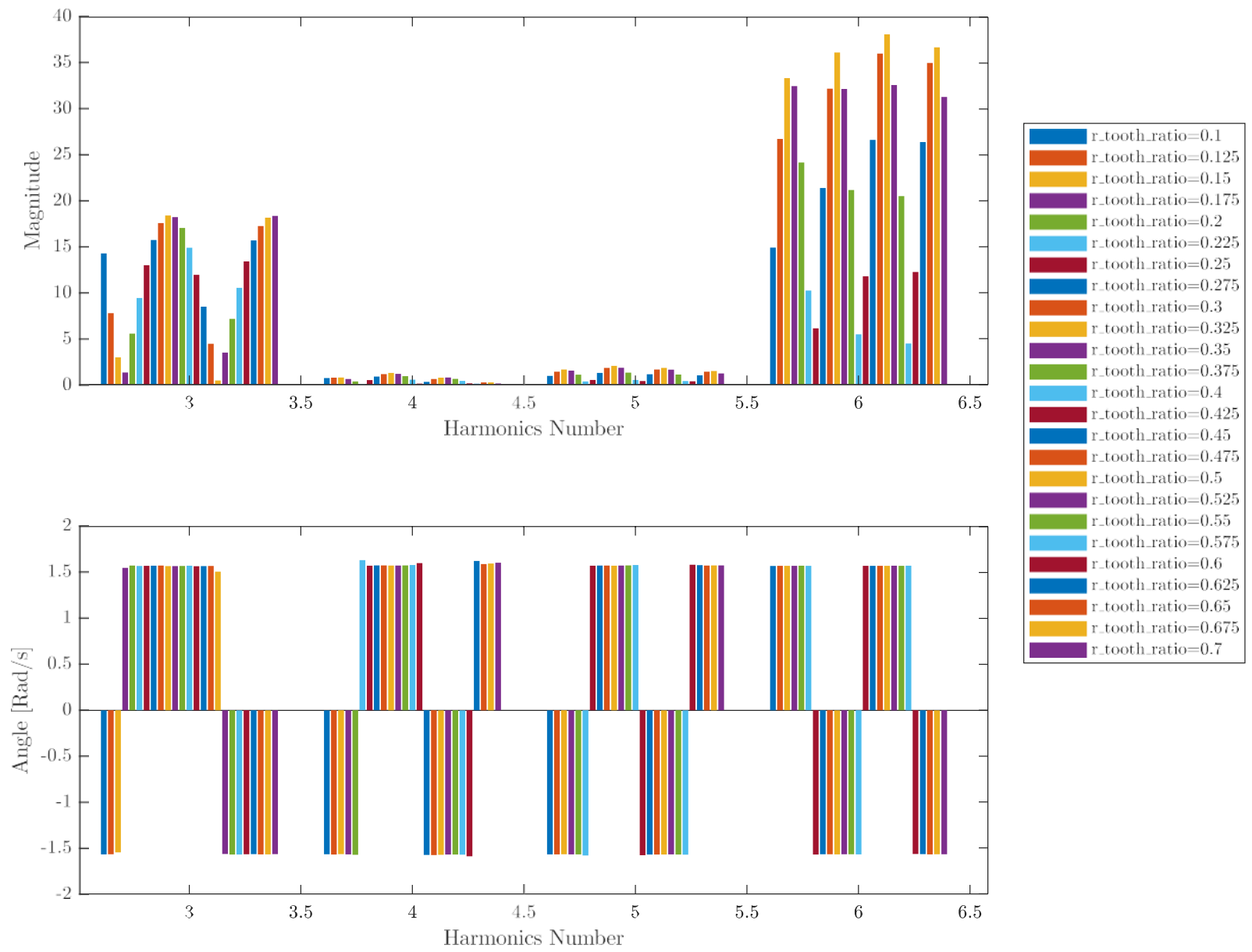

Fig. 11: Cogging torque harmonics across a range of rotor tooth ratios for the FRM.

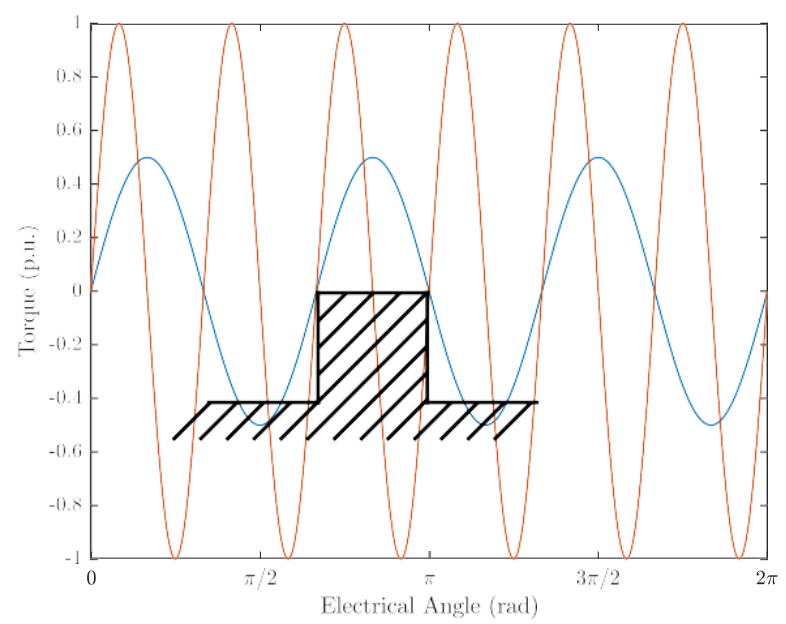

Fig. 12: Example $3^{\text {rd }}$ and $6^{\text {th }}$ harmonics across one electrical cycle with a single tooth drawn on top. variable, with a strong impact on peak voltage and cogging torque. However, the magnet fill ratio was fixed at unity. It is doubtless that a study into variation of the magnet fill ratio would be highly informative and would likely have unpredictable impacts on the peak voltage and cogging torque of the machines.

\section{ACKNOWLEDGMENTS}

This work was supported by the Engineering and Physical Sciences Research Council (grant number EP/S024069/1). simulation work was conducted within the Centre for Doctoral Training in Sustainable Electric Propulsion. The practical work was part funded an InnovateUK smart award, reference 56808 .

\section{REFERENCES}

[1] "Wave energy converter: Mocean pioneering wave technology," Oct 2019. [Online]. Available: https://www.mocean.energy/wave-energy-converter/

[2] T. Whittaker, D. Collier, M. Folley, M. Osterried, A. Henry, and M. Crowley, "The development of oyster - a shallow water surging wave energy converter," in Proceedings of the 7th European wave and tidal energy conference, 2007, pp. 11-14. 
[3] N. J. Baker, M. A. Raihan, A. A. Almoraya, J. W. Burchell, and M. A. Mueller, "Evaluating alternative linear vernier hybrid machine topologies for integration into wave energy converters," IEEE Transactions on Energy Conversion, vol. 33, no. 4, pp. 2007-2017, 2018.

[4] Y. Gao, R. Qu, D. Li, J. Li, and G. Zhou, "Consequent-pole fluxreversal permanent-magnet machine for electric vehicle propulsion," IEEE Transactions on Applied Superconductivity, vol. 26, no. 4, pp. 1-5, 2016.

[5] B. Kim and T. A. Lipo, "Operation and design principles of a pm vernier motor," in 2013 IEEE Energy Conversion Congress and Exposition. IEEE, 2013, pp. 5034-5041.

[6] J. G. Washington, G. J. Atkinson, and N. J. Baker, "Reduction of cogging torque and emf harmonics in modulated pole machines," IEEE Transactions on Energy Conversion, vol. 31, no. 2, pp. 759-768, 2016.

[7] E. Spooner and L. Haydock, "Vernier hybrid machines," IEE ProceedingsElectric Power Applications, vol. 150, no. 6, pp. 655-662, 2003. 